\title{
Study on Leaf Image Feature Extraction of Cucumber Disease
}

\author{
Zhanwu Peng ${ }^{1, a^{*}}$, Xue Wang ${ }^{2, b}$ \\ ${ }^{1} 1$ Information technology teaching and management center, Jilin Agricultural University, Changchun \\ 130118, China \\ ${ }^{2}$ Institute of Scientific and Technical Information of Jilin, Changchun 130021, China \\ a*13908738@qq.com, b21211430@qq.com
}

Keywords: image processing, feature extraction, cucumber disease image

\begin{abstract}
In order to realize the accurate recognition of cucumber disease, feature extraction is a crucial step. The study used computer image processing technology to preprocess and extract features of cucumber disease image, then used professional image acquisition device to obtain the disease image of cucumber, through the preparation of computer software to process image noise reduction and other preprocessing. The research studied the characteristics of cucumber downy mildew, extracted 15 characteristic parameters from three aspects of statistics, shape and color, which will be used as the main basis for disease identification, and improved the accuracy of identification.
\end{abstract}

\section{Introduction}

Crops in the growing process, often by the infection of various diseases, improper handling will cause a decline in yield and quality of agricultural products, if excessive use of pesticides to control not only the agricultural residues of pesticides, even cause the destruction of the ecological environment. Timely and accurate extraction of the characteristics of the disease is a prerequisite for identification of disease, disease prevention and control, [1] this study using computer image processing technology, for agricultural production in the most common infecting cucumber diseases typical cases, downy mildew, research on the feature extraction.

\section{Image Preprocessing}

Using high precision digital cameras and video cameras, optical devices, digital image, eliminating the need for digital image conversion process, although we take all kinds of measures to improve the image quality, but due to the imaging characteristics of optical devices, the resulting image still exist certain dry disturbing factors affecting cucumber downy mildew feature extraction, and the use of digital image processing in the study of some preprocessing algorithms can greatly improve the image of disease forms, creating favorable conditions for accurate extraction of the characteristics of the disease.[2]

Image segmentation processing. Image segmentation is also called threshold segmentation, the purpose is to extract leaf disease position, for each pixel of the input image, gray value in a certain value (threshold T) range, given the output image pixel value is 0 (black), or 255 (white), after the 
transformation, the image becomes to binary image, disease position of the blade and the normal parts to form a larger contrast, so that disease position is extracted.

Image smoothing processing. The image obtained by CCD camera, usually disturbed by noise, the removal of noise in image processing for image smoothing processing, study the $3 \times 3$ neighborhood pixels within the median filtering method to remove image noise, the so-called median filtering method is in the input image $3 \times 3$ neighborhood pixels within, averaged value of the center pixel eight neighborhood as the output image pixel value, through contrast experiment, this method has very obvious effect for reducing noise of disease image samples.

Image edge detection processing. In order to shape features of the images to highlight the disease and the diseases image do edge detection processing, due to the edge of the lesion site was a step like change, so study by a first order differential operator for edge detection.

$f_{x}$ represents the $x$ direction of the differential, $f_{y}$ represents the $y$ direction of the differential.

$$
\left.\begin{array}{ll}
f_{x}=f(x+1, y) & f(x, y) \\
f_{y}=f(x, y+1) & f(x, y)
\end{array}\right)
$$

Edge value $F=\sqrt{f_{x}^{2}+f_{y}^{2}}$, Edge direction $G(x, y)=\left(f_{x}+f_{y}\right)$

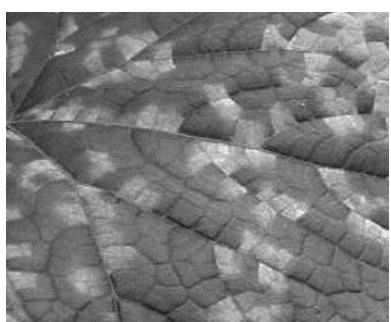

a. Grav image

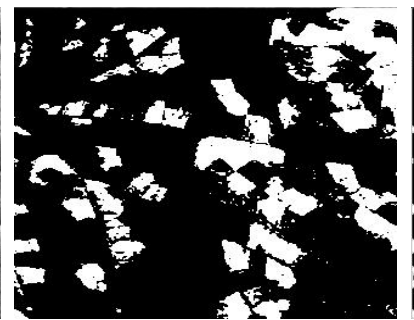

b. Image segmentation processing

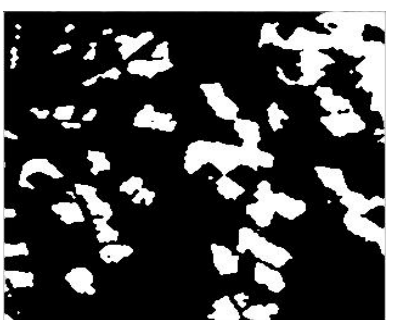

c. Image Smoothing

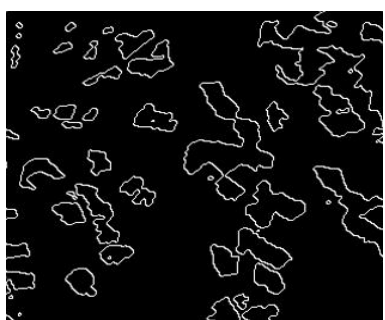

d. Edge detection processing

Fig. 1 Leaves of cucumber downy mildew image preprocessing

\section{Disease feature extraction}

According to the experience of agricultural plant protection experts and consulting relevant literature, we come to the conclusion that the main performance characteristics of Cucumber Downy Mildew: usually the leaf in the middle part of plants and the onset of the disease, and gradually extended to both upper and lower, at the early stage of the disease leaves appear faded green spot and extended to form Brown irregular shaped lesion. Humidity is high in water soaked to the back of the blade, and then produce black mold layer. At the back of the lesion leaves necrosis ooze a colorless or light yellow liquid droplets. The lesion extends quickly, $1 \sim 2$ days due to its expansion by vein and is polygonal.

According to the above characteristics of cucumber downy mildew, we after repeated research trials, from three aspects of spot color, shape and leaf texture extraction disease image characteristic parameters. 
Statistical feature extraction. The statistic feature of image is that it can describe the distribution of gray value of pixels in the image. For cucumber disease leaves, the statistic characteristics can also explain some problems. [3] The first order probability distribution of a gray level image of cucumber disease is defined as: $p(b)=\frac{n(b)}{n}$, Where, $b$ is the pixel gray value, in the range [0 255], $n$ is the total pixels, $n(b)$ is the window of gray value for b number of pixels, and use a graph said called the gray histogram of image and its statistic characteristics include:

Mean value: $\bar{b}=\sum_{b=0}^{L-1} b P(b)$, Mean high gray level image brightness value is also high.

Variance: $\sigma_{b}^{2}=\sum_{b=0}^{L-1}(b-\bar{b})^{2} P(b)$, Variance high gray level image contrast ratio is also high.

Energy: $b_{N}=\sum_{b=0}^{L-1}[P(b)]^{2}$, Entropy: $b_{E}=-\sum_{b=0}^{L-1} P(b) \log [P(b)]$, Energy and entropy reflects the image gray distribution is not uniform, more uneven distribution, image energy value is greater, and entropy is lower.

$$
\text { Moment of inertia: } \omega=\sum_{b=0}^{L-1} b^{2} P(b)
$$

The inertia moment describes the value of the gray level co-occurrence matrix in the image and the local change, and the value of the image is reflected by the change of the texture of the gray image and the definition of the image.

Local stability: $\varepsilon=\sum_{b=0}^{L-1} \frac{P(b)}{1+b^{2}}$

The local variation of image texture is described by local stability, and the value of the image is reflected by the rules of the texture of the blade.

Table1. Leaves of cucumber downy mildew gray statistic characteristics table

\begin{tabular}{ccccccc}
\hline Leaf number & $\bar{b}$ & $\sigma_{b}^{2}$ & $b_{N}$ & $b_{E}$ & $\omega$ & $\varepsilon$ \\
\hline 0 & 102.6582 & 96.2850 & 0.2358 & 1.2789 & 10.0965 & 0.2354 \\
1 & 126.3692 & 26.5420 & 0.1266 & 1.2062 & 11.3620 & 0.6352 \\
2 & 65.0256 & 102.9017 & 0.2390 & 2.5369 & 8.3200 & 0.2150 \\
3 & 223.5620 & 23.5297 & 0.5900 & 2.3325 & 0.9200 & 0.3251 \\
4 & 32.0000 & 158.1282 & 0.3323 & 2.9808 & 3.6521 & 0.6321 \\
5 & 203.9100 & 100.3681 & 0.1005 & 2.1587 & 1.2841 & 0.3205 \\
6 & 30.9682 & 99.0281 & 0.1258 & 1.5680 & 1.2578 & 0.2100 \\
7 & 185.2150 & 153.2760 & 0.6280 & 2.3605 & 5.3621 & 0.6785 \\
8 & 167.3528 & 111.5268 & 0.0658 & 1.2850 & 3.2561 & 0.9600 \\
9 & 133.2100 & 69.3332 & 0.3521 & 1.0095 & 0.2586 & 0.2869 \\
\hline
\end{tabular}


Color feature extraction. Cucumber Leaf Infected with disease, from the color will be significantly changed, so the color feature is an important basis for the identification of diseases. Using color image histogram, to visually describe the distribution of each component of the R, G, B, using a histogram of the column height to the frequency of each pixel in the image representation, ${ }^{[4]}$ due to disease in the sample image each pixel color expressed as $(\mathrm{R}, \mathrm{G}, \mathrm{B})$ a set of values, so the color of each pixel corresponds to $\mathrm{R}, \mathrm{G}, \mathrm{B}$, and $\mathrm{B}$ the diagram of a point, in histogram relation with light and dark point represents the frequency of the appearance of, the brighter the said higher frequency. ${ }^{[5]}$

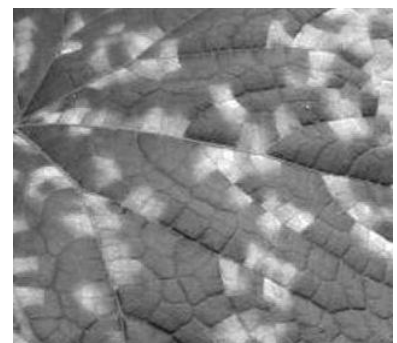

a. Red component

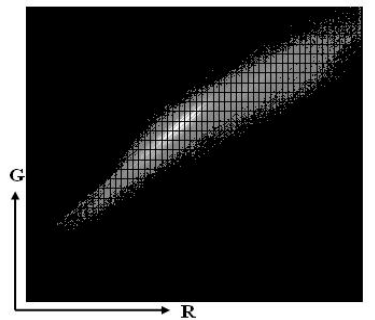

e. $\mathrm{R}$ and $\mathrm{G}$ of the diagram

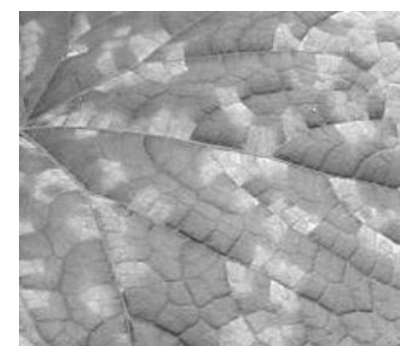

b. Green component

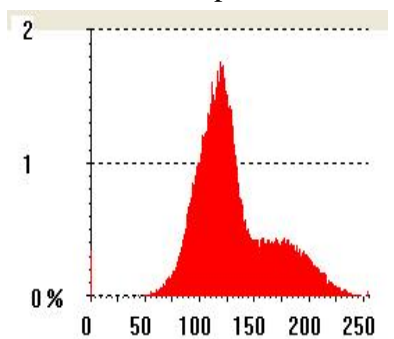

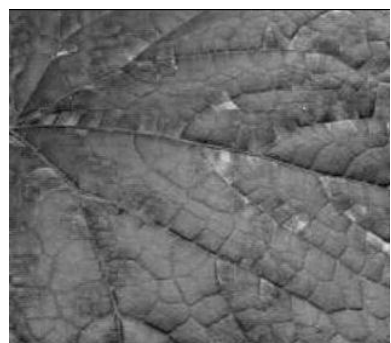

c. Blue component

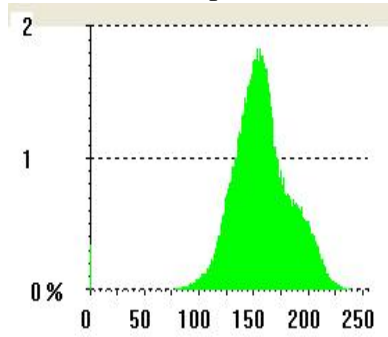

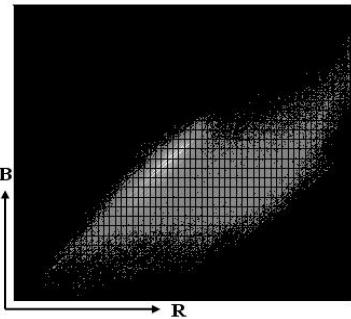

d. $R$ and $B$ of the diagram

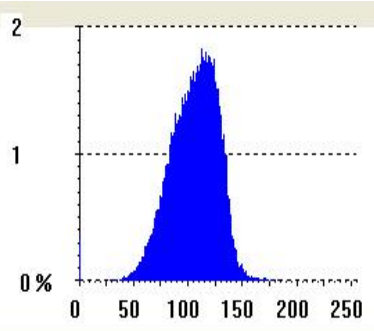

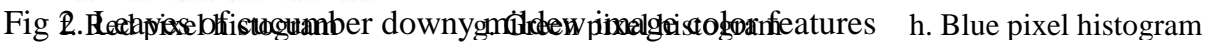

Shape feature extraction. In the leaves of cucumber downy mildew infection. Because the lesion expansion by veins, showing irregular polygon shape feature, after the shape of the lesion after pretreatment is usually surrounded by a closed curve. These irregular shapes of some geometric features can describe the downy mildew of shape feature. ${ }^{[6]}$

Area is $A$, calculate the sum of the pixel values in all closed graphs. $A=\sum_{x=0}^{M} \sum_{y=0}^{N} f(x, y)$

Circumference is $S$, calculate the sum of the distance between the pixels in the closed contour line, $n$ is expressed in this formula chain codes, $c_{i},(i=0,1,2, \ldots, 7)$ is direction of chain code, The length of the vertical or horizontal direction length is 1 , and the code length in the diagonal direction is $\sqrt{2}$.

$$
S=\sum_{i=0}^{n}\left\lfloor 1+\left(\frac{\sqrt{2}-1}{2}\right)\left(1-(-1)^{c_{i}}\right)\right\rfloor
$$

Circularity is $C$, To describe features of the diseased region close to the circular degree, its range is $[0,1]$, The smaller the value of $C$ is closer to the circle, the more deviation from the circle, the more complex the shape. $C=\frac{4 \pi A}{S^{2}}$ 
Center of gravity is $G\left(x_{0}, y_{0}\right)$, the average value of the pixel coordinates of lesion. $G\left(x_{0}, y_{0}\right)=\left(\frac{1}{n} \sum_{i=0}^{n-1} x_{i}, \frac{1}{n} \sum_{i=0}^{n-1} y_{i}\right)$

10 randomly selected lesion leaves, calculating the spot shape parameters, get the following data parameter table:

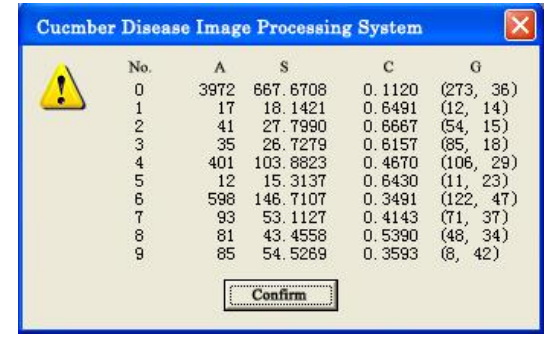

Fig 3. The feature parameters of spot shape of cucumber downy mildew

\section{Conclusion}

1) The current situation of the application of computer image processing technology in crop growth monitoring is analyzed. The method is an important part of precision agriculture production.

2) Taking cucumber downy mildew image as an example, several image preprocessing methods were studied, which created favorable conditions for the accurate extraction of the disease characteristic parameters.

3) According to the case of downy mildew of cucumber, 15 effective features were extracted from three aspects of statistics, color and shape, and the input of the classifier was used as the classifier.

\section{Reference}

[1] Rastogi A, Arora R, Sharma S. Leaf disease detection and grading using computer vision technology \& fuzzy logic[C]// Signal Processing and Integrated Networks (SPIN), 2015 2nd International Conference on. IEEE, 2015:500-505.

[2] Peng-Yun X U, Sun W L, Xia L, et al. Feature Extraction of Spores Based on Digital Image Processing[J]. Journal of Agricultural Mechanization Research, 2008.

[3] Barbedo J G A. Digital image processing techniques for detecting, quantifying and classifying plant diseases.[J]. Springerplus, 2013, 2(1):660-660.

[4] Zhang S W, Shang Y J, Wang L. Plant disease recognition based on plant leaf image[J]. Journal of Animal \& Plant Sciences, 2015, 25(3):42-45.

[5] Barbedo J G A. An Automatic Method to Detect and Measure Leaf Disease Symptoms Using Digital Image Processing[J]. Plant Disease, 2014, 98(98):1709-1716.

[6] Zhou Z E A. Study of Tomato Leaf Diseasa Recognition Based on Computer Vision Technology[J]. Journal of Anhui Agricultural Sciences, 2013. 